6-6-2019

\title{
Measure of Departure from Marginal Average Point-Symmetry for Two-Way Contingency Tables
}

Kiyotaka Iki

Tokyo University of Science, iki@is.noda.tus.ac.jp

Sadao Tomizawa

Tokyo University of Science, tomizawa@is.noda.tus.ac.jp

Follow this and additional works at: https://digitalcommons.wayne.edu/jmasm

Part of the Applied Statistics Commons, Social and Behavioral Sciences Commons, and the Statistical Theory Commons

\section{Recommended Citation}

Iki, T., \& Tomizawa, S. (2018). Measure of departure from marginal average point-symmetry for two-way contingency tables. Journal of Modern Applied Statistical Methods, 17(2), eP2667. doi: 10.22237/jmasm/1559569334

This Regular Article is brought to you for free and open access by the Open Access Journals at DigitalCommons@WayneState. It has been accepted for inclusion in Journal of Modern Applied Statistical Methods by an authorized editor of DigitalCommons@WayneState. 


\section{Measure of Departure from Marginal Average Point-Symmetry for Two-Way Contingency Tables}

\section{Kiyotaka Iki}

Tokyo University of Science

Tokyo, Japan

\author{
Sadao Tomizawa \\ Tokyo University of Science \\ Tokyo, Japan
}

For the analysis of two-way contingency tables with ordered categories, Yamamoto, Tahata, Suzuki, and Tomizawa (2011) considered a measure to represent the degree of departure from marginal point-symmetry. The maximum value of the measure cannot distinguish two kinds of marginal complete asymmetry with respect to the midpoint. A measure is proposed which can distinguish two kinds of marginal asymmetry with respect to the midpoint. It also gives large-sample confidence interval for the proposed measure.

Keywords: Asymmetry, marginal proportional point-symmetry, marginal pointsymmetry, measure, model, ordered category

\section{Introduction}

Consider an $R \times R$ square contingency table with the same row and column classifications. Let $p_{i j}$ denote the probability that an observation will fall in the $i^{\text {th }}$ row and $j^{\text {th }}$ column of the table $(i=1, \ldots, R ; j=1, \ldots, R)$. The symmetry model, which was given by Bowker (1948), is defined by

$$
p_{i j}=p_{j i} \quad(i<j)
$$

This model indicates that the probability that an observation will fall in row category $i$ and column category $j$ is equal to the probability that the observation falls in row category $j$ and column category $i$. Namely, this describes a structure of symmetry of the cell probabilities $\left\{p_{i j}\right\}$ with respect to the main diagonal of the table. For the symmetry model see also Bishop, Fienberg, and Holland (1975, p.

doi: 10.22237/jmasm/1559569334 | Accepted: December 9, 2017; Published: June 6, 2019.

Correspondence: Kiyotaka Iki, iki@is.noda.tus.ac.jp 


\section{IKI \& TOMIZAWA}

282), Caussinus (1965), McCullagh (1978), Goodman (1979), Agresti (2002, p. 424), Tomizawa and Tahata (2007), and Tahata and Tomizawa (2014).

The marginal homogeneity (or marginal symmetry) model is defined by

$$
p_{i \cdot}=p_{\cdot i} \quad(i=1, \ldots, R)
$$

where

$$
p_{i \cdot}=\sum_{t=1}^{R} p_{i t}, \quad p_{\cdot i}=\sum_{s=1}^{R} p_{s i}
$$

see, e.g., Stuart (1955), Bhapkar (1966), Bishop et al. (1975, p. 282), Tomizawa and Tahata (2007), and Tahata and Tomizawa (2014). The marginal homogeneity model indicates that the row marginal distribution is identical to the column marginal distribution.

Wall and Lienert (1976) considered the point-symmetry model, defined by

$$
p_{i j}=p_{i^{*} j^{*}} \quad(i=1, \ldots, R ; j=1, \ldots, R)
$$

where the symbol $*$ denotes $i^{*}=R+1-i$.

Consider an $R \times C$ rectangular contingency table with ordered categories. Let $p_{i j}$ denote the probability that an observation will fall in the $i^{\text {th }}$ row and $j^{\text {th }}$ column of the table $(i=1, \ldots, R ; j=1, \ldots, C)$. Tomizawa (1985) extended the pointsymmetry model for an $R \times C$ contingency table as follows:

$$
p_{i j}=p_{i^{*} j^{* *}} \quad(i=1, \ldots, R ; j=1, \ldots, C)
$$

where $i^{*}=R+1-i$ and $j^{* *}=C+1-j$. This model indicates that the probability that an observation will fall in row category $i$ and column category $j$ is equal to the probability that the observation falls in row category $i^{*}$ and column category $j^{* *}$. Namely, this describes a structure of point-symmetry of cell probabilities with respect to the center cell or center point in the table. Also see Tomizawa and Tahata (2007) and Tahata and Tomizawa (2014). Tomizawa (1985) also considered the marginal point-symmetry model, defined by

$$
p_{i .}=p_{i^{*} .} \quad(i=1, \ldots, R)
$$




\section{MEASURE OF MARGINAL AVERAGE POINT-SYMMETRY}

and

$$
p_{\cdot j}=p_{\cdot j^{* *}} \quad(j=1, \ldots, C) .
$$

This model indicates that the row marginal distribution is point-symmetric with respect to the midpoint and the column marginal distribution is also pointsymmetric with respect to the midpoint. Let [ ] denote the Gauss symbol (i.e., for real number $x,[x]$ denotes the maximum integer which is not larger than $x$ ). For example, when $R=4,[R / 2]=2$, and when $R=5,[R / 2]=2$. The marginal pointsymmetry model is also expressed as essentially

$$
p_{i .}=p_{i^{*} .} \quad\left(i=1, \ldots,\left[\frac{R}{2}\right]\right)
$$

and

$$
p_{\cdot j}=p_{\cdot j^{* *}}\left(j=1, \ldots,\left[\frac{C}{2}\right]\right) .
$$

When the model does not hold, there is also interested in measuring the degree of departure from the model. For the measures to represent the degree of departure from the symmetry, the point-symmetry and the marginal homogeneity models, see, e.g., Tomizawa (1994, 1995), Tomizawa, Seo, and Yamamoto (1998), Tomizawa, Miyamoto, and Hatanaka (2001), and Tomizawa, Yamamoto, and Tahata (2007). For the measure from the marginal point-symmetry model, Yamamoto, Tahata, Suzuki, and Tomizawa (2011) proposed the power-divergence type measure $\psi^{(\lambda)}$; see Appendix 1. However, when the measure $\psi^{(\lambda)}$ equals 1, it is not possible to distinguish two kinds of row (column) complete asymmetry with respect to the

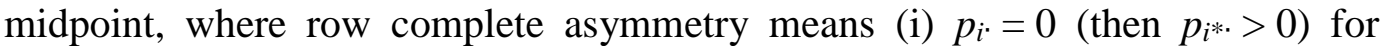
$i=1, \ldots,[R / 2]$, or (ii) $p_{i^{*}}=0$ (then $p_{i}>0$ ) for $i=1, \ldots,[R / 2]$, and column complete asymmetry means (i) $p \cdot j=0$ (then $p \cdot j^{* *}>0$ ) for $j=1, \ldots,[C / 2]$, or (ii) $p \cdot j^{* *}=0$ (then $p \cdot j>0)$ for $j=1, \ldots,[C / 2]$. Because these two kinds of row (column) complete asymmetry indicate the opposite different maximum departures from marginal point-symmetry with respect to the midpoint, the interest is in proposing a measure which can take the different values for them.

The purpose of present study is to propose a measure which can distinguish two kinds of the marginal complete asymmetry with respect to the midpoint for 


\section{IKI \& TOMIZAWA}

rectangular contingency tables with ordered categories. The measure lies between -1 and 1 although the measure $\psi^{(\lambda)}$ lies between 0 and 1 , and it may be useful for comparing the degree of departure from marginal point-symmetry in several tables.

\section{Measure}

Consider the $R \times C$ contingency table. Let

$$
\begin{aligned}
& \delta_{1}=\sum_{i=1}^{[R / 2]}\left(p_{i \cdot}+p_{i^{*} .}\right), \quad \delta_{2}=\sum_{j=1}^{[C / 2]}\left(p_{\cdot j}+p_{\cdot j^{* *}}\right), \\
& q_{i \cdot}=\frac{p_{i \cdot}}{\delta_{1}}, \quad q_{i^{*} \cdot}=\frac{p_{i^{*} \cdot}}{\delta_{1}} \quad\left(i=1, \ldots,\left[\frac{R}{2}\right]\right),
\end{aligned}
$$

and

$$
q_{\cdot j}=\frac{p_{\cdot j}}{\delta_{2}}, \quad q_{\cdot j^{* *}}=\frac{p_{\cdot j^{* *}}}{\delta_{2}}, \quad\left(j=1, \ldots,\left[\frac{C}{2}\right]\right) .
$$

Assuming $\left\{p_{i^{\cdot}}+p_{\left.i^{*} \cdot \neq 0\right\}}\right.$ and $\left\{p \cdot j+p \cdot *^{* *} \neq 0\right\}$, a measure is proposed to represent the degree of departure from marginal point-symmetry, defined by

$$
\varphi_{\mathrm{MPS}}=\frac{\delta_{1} \varphi_{1}+\delta_{2} \varphi_{2}}{\delta_{1}+\delta_{2}}
$$

where

$$
\begin{aligned}
\varphi_{1} & =\frac{4}{\pi} \sum_{i=1}^{[R / 2]}\left(q_{i \cdot}+q_{i^{*} \cdot}\right)\left(\theta_{1(i)}-\frac{\pi}{4}\right), \\
\theta_{1(i)} & =\cos ^{-1}\left(\frac{q_{i \cdot}}{\sqrt{q_{i \cdot}^{2}+q_{i^{*}}^{2}}}\right), \\
\varphi_{2} & =\frac{4}{\pi} \sum_{j=1}^{[C / 2]}\left(q_{\cdot j}+q_{\cdot j^{* *}}\right)\left(\theta_{2(j)}-\frac{\pi}{4}\right),
\end{aligned}
$$




\section{MEASURE OF MARGINAL AVERAGE POINT-SYMMETRY}

$$
\theta_{2(j)}=\cos ^{-1}\left(\frac{q_{\cdot j}}{\sqrt{q_{\cdot j}^{2}+q_{\cdot j^{* * *}}^{2}}}\right) \text {. }
$$

The submeasure $\varphi_{1}$ represents the degree of departure from point-symmetry of row marginal distribution and the submeasure $\varphi_{2}$ represents the degree of departure from point-symmetry of column marginal distribution. The measure $\varphi_{\text {MPS }}$, being the weighted sum of the submeasures $\varphi_{1}$ and $\varphi_{2}$, represents the degree of departure from marginal point-symmetry.

The ranges of $\left\{\theta_{1(i)}\right\}$ and $\left\{\theta_{2(j)}\right\}$ are $0 \leq \theta_{1(i)} \leq \pi / 2$ and $0 \leq \theta_{2(j)} \leq \pi / 2$. The submeasures $\varphi_{1}$ and $\varphi_{2}$ lie between -1 and 1 . Therefore, the measure $\varphi_{\text {MPS }}$ lies between -1 and 1 . The submeasure $\varphi_{1}$ has characteristics that (i) $\varphi_{1}=1$ if and only if $p_{i}=0$ (then $p_{i^{*}}>0$ ) for $i=1, \ldots,[R / 2]$, say, row upper complete asymmetry with respect to the midpoint, and (ii) $\varphi_{1}=-1$ if and only if $p_{i^{*}}=0$ (then $p_{i}>0$ ) for $i=1, \ldots,[R / 2]$, say, row lower complete asymmetry with respect to the midpoint. Similarly, the submeasure $\varphi_{2}$ has characteristics that (i) $\varphi_{2}=1$ if and only if $p_{\cdot j}=0$ (then $p \cdot *^{*}>0$ ) for $j=1, \ldots,[C / 2]$, say, column upper complete asymmetry with respect to the midpoint, and (ii) $\varphi_{2}=-1$ if and only if $p \cdot \cdot^{* *}=0$ (then $p \cdot j>0$ ) for $j=1, \ldots,[C / 2]$, say, column lower complete asymmetry with respect to the midpoint. The measure $\varphi_{\text {MPS }}$ has characteristics that (i) $\varphi_{\text {MPS }}=1$ if and only if $\varphi_{1}=\varphi_{2}=1$, and (ii) $\varphi_{\text {MPS }}=-1$ if and only if $\varphi_{1}=\varphi_{2}=-1$.

The submeasure $\varphi_{1}=0$ indicates the average of $\left\{\theta_{1(i)}-(\pi / 4)\right\}, i=1, \ldots,[R / 2]$, equals zero. When $\varphi_{1}=0$, this structure is referred to as the row average pointsymmetry. Similarly, when $\varphi_{2}=0$, this structure is referred to as the column average point-symmetry. If the marginal point-symmetry model holds then the row (column) average point-symmetry holds; but the converse does not. Using the submeasure $\varphi_{1}\left(\varphi_{2}\right)$, it can be deteremined whether the row (column) average pointsymmetry departs toward the row (column) upper complete asymmetry with respect to the midpoint or toward the row (column) lower complete asymmetry with respect to the midpoint. When $\varphi_{\text {MPS }}=0$, this structure will be referred to as the marginal average point-symmetry. We note that if the marginal point-symmetry model holds then the marginal average point-symmetry holds; but the converse does not. However, it is difficult to consider the exact interpretation of the marginal average point-symmetry. If the submeasures $\varphi_{1}$ and $\varphi_{2}$ equal 0 then $\varphi_{\text {MPs }}$ equals 0 , but the converse does not hold.

For example, consider the artificial probabilities in Table 1. For Table 1a, since there are the structures of row upper complete asymmetry with respect to the midpoint (i.e., $\varphi_{1}=1$ ) and column upper complete asymmetry with respect to the 


\section{IKI \& TOMIZAWA}

midpoint (i.e., $\varphi_{2}=1$ ), we see that the measure $\varphi_{\text {MPS }}=1$. Also, for Table $1 \mathrm{~b}$, because there are the structures of row lower complete asymmetry (i.e., $\varphi_{1}=-1$ ) and column lower complete asymmetry (i.e., $\varphi_{2}=-1$ ), we see that the measure $\varphi_{\text {MPS }}=-1$. For Table 1c, because there are the structures of row lower complete asymmetry (i.e., $\varphi_{1}=-1$ ) and column upper complete asymmetry (i.e., $\varphi_{2}=1$ ), we see that the measure $\varphi_{\text {MPS }}=0$.

\section{Relationship between Measure and Model}

Consider the relationship between the measure $\varphi_{\text {MPs }}$ and a model. Define the model by

$$
p_{i .}=\Delta_{1} p_{i^{*} .} \quad\left(i=1, \ldots,\left[\frac{R}{2}\right]\right)
$$

Table 1. Artificial probabilities

\begin{tabular}{rrrrrr} 
(a) & \multicolumn{5}{c}{$\boldsymbol{Y}$} \\
\cline { 2 - 5 } $\boldsymbol{X}$ & $\mathbf{( 1 )}$ & $\mathbf{( 2 )}$ & $\mathbf{( 3 )}$ & $\mathbf{( 4 )}$ & Total \\
\hline$(1)$ & 0.0 & 0.0 & 0.0 & 0.0 & 0.0 \\
$(2)$ & 0.0 & 0.0 & 0.0 & 0.0 & 0.0 \\
$(3)$ & 0.0 & 0.0 & 0.3 & 0.2 & 0.5 \\
$(4)$ & 0.0 & 0.0 & 0.2 & 0.3 & 0.5 \\
Total & 0.0 & 0.0 & 0.5 & 0.5 & 1.0 \\
\hline
\end{tabular}

\begin{tabular}{rrrrrr} 
(b) & \multicolumn{5}{c}{$\boldsymbol{Y}$} \\
\cline { 2 - 5 } $\boldsymbol{X}$ & $\mathbf{( 1 )}$ & $\mathbf{( 2 )}$ & $\mathbf{( 3 )}$ & $\mathbf{( 4 )}$ & Total \\
\hline$(1)$ & 0.3 & 0.2 & 0.0 & 0.0 & 0.5 \\
$(2)$ & 0.2 & 0.3 & 0.0 & 0.0 & 0.5 \\
$(3)$ & 0.0 & 0.0 & 0.0 & 0.0 & 0.0 \\
$(4)$ & 0.0 & 0.0 & 0.0 & 0.0 & 0.0 \\
Total & 0.5 & 0.5 & 0.0 & 0.0 & 1.0 \\
\hline
\end{tabular}

\begin{tabular}{rrrrrr}
\multicolumn{5}{c}{$\boldsymbol{Y}$} \\
$\boldsymbol{n y y y y n} \boldsymbol{X}$ & $\mathbf{( 1 )}$ & $\mathbf{( 2 )}$ & $\mathbf{( 3 )}$ & $\mathbf{( 4 )}$ & Total \\
\hline$(1)$ & 0.0 & 0.0 & 0.3 & 0.2 & 0.5 \\
$(2)$ & 0.0 & 0.0 & 0.2 & 0.3 & 0.5 \\
$(3)$ & 0.0 & 0.0 & 0.0 & 0.0 & 0.0 \\
$(4)$ & 0.0 & 0.0 & 0.0 & 0.0 & 0.0 \\
Total & 0.0 & 0.0 & 0.5 & 0.5 & 1.0 \\
\hline
\end{tabular}




\section{MEASURE OF MARGINAL AVERAGE POINT-SYMMETRY}

and

$$
p_{\cdot j}=\Delta_{2} p_{\cdot j^{* *}} \quad\left(j=1, \ldots,\left[\frac{C}{2}\right]\right),
$$

where $\Delta_{1}$ and $\Delta_{2}$ are unspecified. This structure is referred to as the marginal proportional point-symmetry model. A special case of this model obtained by setting $\Delta_{1}=\Delta_{2}=1$ is the marginal point-symmetry model. If there is a structure of the marginal proportional point-symmetry model in the table, then the submeasures $\varphi_{1}$ and $\varphi_{2}$ can be simply (as a function of parameters $\Delta_{1}$ and $\Delta_{2}$, respectively) expressed as

$$
\varphi_{1}=\frac{4}{\pi} \cos ^{-1}\left(\frac{\Delta_{1}}{\sqrt{\Delta_{1}^{2}+1}}\right)-1
$$

and

$$
\varphi_{2}=\frac{4}{\pi} \cos ^{-1}\left(\frac{\Delta_{2}}{\sqrt{\Delta_{2}^{2}+1}}\right)-1 .
$$

Under the marginal proportional point-symmetry model, $\varphi_{1}=\varphi_{2}=0$ if and only if the marginal point-symmetry model holds, i.e., $\Delta_{1}=\Delta_{2}=1$. As the value of $\Delta_{1}\left(\Delta_{2}\right)$ approaches zero, the submeasure $\varphi_{1}\left(\varphi_{2}\right)$ approaches 1 , and as the value of $\Delta_{1}\left(\Delta_{2}\right)$ approaches infinity, the submeasure $\varphi_{1}\left(\varphi_{2}\right)$ approaches -1 . Denote $\varphi_{1}$ and $\varphi_{2}$ by $\varphi_{1}\left(\Delta_{1}\right)$ and $\varphi_{2}\left(\Delta_{2}\right)$, respectively. Let

$$
\theta_{1}=\cos ^{-1}\left(\frac{\Delta_{1}}{\sqrt{\Delta_{1}^{2}+1}}\right)
$$

Noting $\varphi_{1}\left(\Delta_{1}\right)=4 \theta_{1} / \pi-1$, 


$$
\begin{aligned}
\varphi_{1}\left(\frac{1}{\Delta_{1}}\right) & =\frac{4}{\pi} \cos ^{-1}\left(\frac{\frac{1}{\Delta_{1}}}{\sqrt{\frac{1}{\Delta_{1}}+1}}\right)-1 \\
& =\frac{4}{\pi} \cos ^{-1}\left(\frac{1}{\sqrt{1+\Delta_{1}^{2}}}\right)-1 \\
& =\frac{4}{\pi}\left(\frac{\pi}{2}-\theta_{1}\right)-1 \\
& =-\varphi_{1}\left(\Delta_{1}\right)
\end{aligned}
$$

Thus, $\left|\varphi_{1}\left(\Delta_{1}\right)\right|$ equals $\left|\varphi_{1}\left(1 / \Delta_{1}\right)\right|$. Similarly, $\left|\varphi_{2}\left(\Delta_{2}\right)\right|$ equals $\left|\varphi_{2}\left(1 / \Delta_{2}\right)\right|$. For comparisons between several tables, if it can be estimated that there is a structure of the marginal proportional point-symmetry model in each table, then the measure $\varphi_{\text {MPS }}$ would be adequate for representing and comparing the degree of departure from the marginal point-symmetry model.

\section{Approximate Confidence Interval for Measures}

Let $n_{i j}$ denote the observed frequency in the $i^{\text {th }}$ row and $j^{\text {th }}$ column of the table $(i=1, \ldots, R ; j=1, \ldots, C)$. Assuming that a multinomial distribution applies to the $R \times C$ table, consider an approximate standard error and large-sample confidence interval for the measure $\varphi_{\text {MPS }}$ and the submeasures $\varphi_{1}$ and $\varphi_{2}$ using the delta method, descriptions of which are given by, for example, Bishop et al. (1975, Sec. 14.6). The sample version of $\varphi_{\text {MPS }}, \hat{\varphi}_{\text {MPS }}$, is given by $\varphi_{\text {MPS }}$ with $\left\{p_{i j}\right\}$ replaced by $\left\{\hat{p}_{i j}\right\}$, where $\hat{p}_{i j}=n_{i j} / n$ and $n=\Sigma \Sigma n_{i j}$. Using the delta method, $\sqrt{n}\left(\hat{\varphi}_{\mathrm{MPS}}-\varphi_{\mathrm{MPS}}\right)$ has asymptotically (as $n \rightarrow \infty$ ) a normal distribution with mean zero and variance

$$
\begin{gathered}
\sigma^{2}\left[\varphi_{\mathrm{MPS}}\right]=\sum_{i=1}^{R} \sum_{j=1}^{C} p_{i j}\left(\frac{\partial \varphi_{\mathrm{MPS}}}{\partial p_{i j}}\right)^{2}, \\
\frac{\partial \varphi_{\mathrm{MPS}}}{\partial p_{i j}}=\frac{1}{\left(\delta_{1}+\delta_{2}\right)^{2}}\left\{\left(\delta_{1}+\delta_{2}\right)\left(\frac{\partial \varphi_{1}}{\partial p_{i j}} \delta_{1}+\frac{\partial \varphi_{2}}{\partial p_{i j}} \delta_{2}\right)+\left(\varphi_{1}-\varphi_{2}\right)\left(\frac{\partial \delta_{1}}{\partial p_{i j}} \delta_{2}-\frac{\partial \delta_{2}}{\partial p_{i j}} \delta_{1}\right)\right\}
\end{gathered}
$$




\section{MEASURE OF MARGINAL AVERAGE POINT-SYMMETRY}

$$
\begin{aligned}
& \frac{\partial \delta_{1}}{\partial p_{i j}}= \begin{cases}1 & i=1, \ldots,\left[\frac{R}{2}\right],\left[\frac{R+1}{2}\right]+1, \ldots, R ; j=1, \ldots, C, \\
0 & \text { otherwise }\end{cases} \\
& \frac{\partial \delta_{2}}{\partial p_{i j}}= \begin{cases}1 & i=1, \ldots, R ; j=1, \ldots,\left[\frac{C}{2}\right],\left[\frac{C+1}{2}\right]+1, \ldots, C, \\
0 & \text { otherwise }\end{cases} \\
& \frac{\partial \varphi_{1}}{\partial p_{i j}}= \begin{cases}\frac{4}{\pi \delta_{1}}\left\{\cos ^{-1}\left(\frac{p_{i \cdot}}{\sqrt{p_{i \cdot}^{2}+p_{i^{*} \cdot}^{2}}}\right)-\frac{p_{i^{*} \cdot}\left(p_{i^{*}}+p_{i^{*}}\right)}{p_{i^{*}}^{2}+p_{i^{*} \cdot}^{2}}\right\}-\frac{\varphi_{1}+1}{\delta_{1}} & i=1, \ldots,\left[\frac{R}{2}\right], \\
\frac{4}{\pi \delta_{1}}\left\{\cos ^{-1}\left(\frac{p_{i^{*} \cdot}}{\sqrt{p_{i \cdot}^{2}+p_{i^{*}}^{2}}}\right)+\frac{p_{i^{*} \cdot}\left(p_{i^{*}}+p_{i^{*}}\right)}{p_{i \cdot}^{2}+p_{i^{*} \cdot}^{2}}\right\}-\frac{\varphi_{1}+1}{\delta_{1}} & i=\left[\frac{R+1}{2}\right]+1, \ldots, R, \\
0 & \text { otherwise }\end{cases} \\
& \frac{\partial \varphi_{2}}{\partial p_{i j}}= \begin{cases}\frac{4}{\pi \delta_{2}}\left\{\cos ^{-1}\left(\frac{p_{\cdot j}}{\sqrt{p_{\cdot j}^{2}+p_{\cdot j^{*}}^{2}}}\right)-\frac{p_{\cdot j^{*}}\left(p_{\cdot j}+p_{\cdot j^{*}}\right.}{p_{\cdot j}^{2}+p_{\cdot j^{*}}^{2}}\right\}-\frac{\varphi_{2}+1}{\delta_{2}} & j=1, \ldots,\left[\frac{C}{2}\right], \\
\frac{4}{\pi \delta_{2}}\left\{\cos ^{-1}\left(\frac{p_{j^{* *}}}{\sqrt{p_{\cdot j}^{2}+p_{\cdot j^{*}}^{2}}}\right)+\frac{p_{\cdot j^{*}}\left(p_{\cdot j}+p_{\cdot j^{*}}\right.}{p_{\cdot j}^{2}+p_{\cdot j^{* *}}^{2}}\right\}-\frac{\varphi_{2}+1}{\delta_{2}} & j=\left[\frac{C+1}{2}\right]+1, \ldots, C, \\
0 & \text { otherwise }\end{cases}
\end{aligned}
$$

Let $\hat{\sigma}^{2}\left[\varphi_{\mathrm{MPS}}\right]$ denote $\sigma^{2}\left[\varphi_{\mathrm{MPS}}\right]$ with $\left\{p_{i j}\right\}$ replaced by $\left\{\hat{p}_{i j}\right\}$. Then $\hat{\sigma}\left[\varphi_{\mathrm{MPS}}\right] / \sqrt{n}$ is an estimated approximate standard error of $\hat{\varphi}_{\mathrm{MPS}}$, and

$$
\hat{\varphi}_{\mathrm{MPS}} \pm z_{\alpha / 2} \hat{\sigma}\left[\varphi_{\mathrm{MPS}}\right] / \sqrt{n}
$$

is an approximate $100(1-\alpha) \%$ confidence interval for $\varphi_{\mathrm{MPS}}$, where $z_{\alpha / 2}$ is the percentage point from the standard normal distribution corresponding to a two-tail probability of $\alpha$.

Similarly, for $k=1,2, \sqrt{n}\left(\hat{\varphi}_{k}-\varphi_{k}\right)$ has asymptotically (as as $\left.n \rightarrow \infty\right)$ a normal distribution with mean zero and variance 


$$
\sigma^{2}\left[\varphi_{\mathrm{k}}\right]=\sum_{i=1}^{R} \sum_{j=1}^{C} p_{i j}\left(\frac{\partial \varphi_{\mathrm{k}}}{\partial p_{i j}}\right)^{2}
$$

and

$$
\hat{\varphi}_{k} \pm z_{\alpha / 2} \hat{\sigma}\left[\varphi_{k}\right] / \sqrt{n}
$$

is an approximate $100(1-\alpha) \%$ confidence interval for $\varphi_{k}$.

\section{Example}

Consider the data in Table 2 taken from Agresti (2002, p. 462). They are the results of a randomized double-blind clinical trial comparing an active hypnotic drug with a placebo in patients with insomnia. The outcome variable is patient's reported time to fall asleep, measured using 4 categories ( $<20$ minutes, 20-30 minutes, 30-60 minutes, and $>60$ minutes).

From Table $3 \mathrm{a}$, for the data in Table $2 \mathrm{a}$, the estimated value of the submeasure $\varphi_{1}$ is $0.545(>0)$, and the confidence interval for $\varphi_{1}$ does not include zero. These would indicate patient's reported time before treatment in the Active treatment group is estimated to be 54.5 percent of the maximum departure toward the row upper complete asymmetry with respect to the midpoint.

Table 2. Insomniac patient's reported time (in minutes) to fall asleep after going to bed, from from Agresti (2002, p. 462)

\begin{tabular}{rrrrrr} 
(a) & \multicolumn{4}{c}{ Follow-up } & \\
\cline { 2 - 5 } Initial & $<\mathbf{2 0}$ & $\mathbf{2 0 - 3 0}$ & $\mathbf{3 0 - 6 0}$ & $\mathbf{7 0}$ & Total \\
\hline$<20$ & 7 & 4 & 1 & 0 & 12 \\
$20-30$ & 11 & 5 & 2 & 2 & 20 \\
$30-60$ & 13 & 23 & 3 & 1 & 40 \\
$>60$ & 9 & 17 & 13 & 8 & 47 \\
Total & 40 & 49 & 19 & 11 & 119 \\
\hline
\end{tabular}

\begin{tabular}{rrrrrr} 
(b) & \multicolumn{4}{c}{ Follow-up } & \\
\cline { 2 - 5 } Initial & $<\mathbf{2 0}$ & $\mathbf{2 0 - 3 0}$ & $\mathbf{3 0 - 6 0}$ & $>\mathbf{6 0}$ & Total \\
\hline$<20$ & 7 & 4 & 2 & 1 & 14 \\
$20-30$ & 14 & 5 & 1 & 0 & 20 \\
$30-60$ & 6 & 9 & 18 & 2 & 35 \\
$>60$ & 4 & 11 & 14 & 22 & 51 \\
Total & 31 & 29 & 35 & 25 & 120 \\
\hline
\end{tabular}




\section{MEASURE OF MARGINAL AVERAGE POINT-SYMMETRY}

Table 3. The estimated measures, their estimated approximate standard errors, and approximate $95 \%$ confidence intervals for measures, applied to (a) Table $2 a$ and (b) Table $2 b$

(a)

\begin{tabular}{rrrr} 
Measure & Estimated measure & Standard error & Confidence interval \\
\hline$\varphi_{1}$ & 0.545 & 0.087 & $(0.375,0.714)$ \\
$\varphi_{2}$ & -0.584 & 0.082 & $(-0.745,-0.424)$ \\
$\varphi_{\mathrm{MPS}}$ & -0.020 & 0.063 & $(-0.143,0.103)$ \\
$\psi_{1}^{(0)}$ & 0.176 & 0.060 & $(0.058,0.294)$ \\
$\psi_{2}^{(0)}$ & 0.189 & 0.063 & $(0.662,0.312)$ \\
$\psi^{(0)}$ & 0.182 & 0.038 & $(0.107,0.258)$ \\
\hline
\end{tabular}

(b)

\begin{tabular}{rrrr} 
Measure & Estimated measure & Standard error & Confidence interval \\
\hline$\varphi_{1}$ & 0.512 & 0.089 & $(0.337,0.688)$ \\
$\varphi_{2}$ & 0.000 & 0.115 & $(-0.226,0.226)$ \\
$\varphi_{M P S}$ & 0.256 & 0.088 & $(0.083,0.429)$ \\
$\psi_{1}^{(0)}$ & 0.159 & 0.058 & $(0.047,0.272)$ \\
$\psi_{2}^{(0)}$ & 0.007 & 0.013 & $(-0.019,0.033)$ \\
$\psi^{(0)}$ & 0.083 & 0.028 & $(0.029,0.138)$ \\
\hline
\end{tabular}

Also, from Table 3a, the estimated value of the submeasure $\varphi_{2}$ is $-0.584(<0)$, and the confidence interval for $\varphi_{2}$ does not include zero. These would indicate patients' reported time after treatment in the Active treatment group is estimated to be $58.4 \%$ of the maximum departure toward the column lower complete asymmetry with respect to the midpoint. Because the absolute values of submeasures $\varphi_{1}$ and $\varphi_{2}$ are almost the same, the measure $\varphi_{\text {MPS }}$ is estimated to be close to zero, and the confidence interval for $\varphi_{\text {MPS }}$ includes zero. However, the estimated values of $\psi_{1}^{(0)}$ and $\psi_{2}^{(0)}$ are 0.176 and 0.189 , respectively. Because these values are almost the same, the estimated value of the measure $\psi^{(0)}$ is also close to $\psi_{1}^{(0)}$ and $\psi_{2}^{(0)}$.

From Table $3 b$, for the data in Table $2 b$ the estimated value of the submeasure $\varphi_{1}$ is $0.512(>0)$, and the confidence interval for $\varphi_{1}$ does not include zero. These indicates patients' reported time before treatment in the Placebo treatment group is estimated to be $51.2 \%$ of the maximum departure toward the row upper complete asymmetry with respect to the midpoint. From Table $3 b$, the estimated value of the submeasure $\varphi_{2}$ is 0.000 , and the confidence interval for $\varphi_{2}$ includes zero. These indicates there is a structure of the column average point-symmetry for patient's reported time after treatment in the Placebo treatment group. The estimated values 
of the measures $\psi_{1}^{(0)}, \psi_{2}^{(0)}$, and $\psi^{(0)}$ show a similar trend with the estimated values of the measures $\varphi_{1}, \varphi_{2}$, and $\varphi_{\mathrm{MPS}}$, respectively.

In addition, when the data in Tables $2 \mathrm{a}$ and $2 \mathrm{~b}$ are compared using the estimated submeasure $\varphi_{1}$, the degree of departure toward the row upper complete asymmetry with respect to the midpoint is almost same for the data in Tables $2 \mathrm{a}$ and 2 b. However, when comparing using the estimated submeasure $\varphi_{2}$, for patients' reported time after treatment, the degree of departure toward the column lower complete asymmetry with respect to the midpoint is greater in the Active treatment than in the Placebo treatment. Therefore, patients' reported time after treatment in the Active treatment would tend to be shorter than that in the Placebo treatment. These interpretation cannot be obtained by using the measures $\psi_{1}^{(0)}, \psi_{2}^{(0)}$, and $\psi^{(0)}$.

\section{Conclusion}

The measure $\varphi_{\text {MPS }}$ and submeasures $\varphi_{1}$ and $\varphi_{2}$ can distinguish two kinds of complete asymmetry with respect to the midpoint although the measure $\psi^{(\lambda)}$ in Yamamoto et al. (2011) cannot distinguish them. Because the measure $\varphi_{\text {MPs }}$ lies between -1 and 1 (although the measure $\psi^{(\lambda)}$ lies between 0 and 1) without the dimension and the sample size, $\varphi_{\text {MPS }}$ is useful for comparing the degrees of departure from marginal average point-symmetry in several tables. When the marginal proportional pointsymmetry model holds, the measure $\varphi_{\text {MPS }}$ are represented by two parameters: $\Delta_{1}$ and $\Delta_{2} .\left|\varphi_{\text {MPS }}\right|$ increases as the value of $\Delta_{1}\left(\Delta_{2}\right)$ approaches zero or infinity from 1. Therefore, $\varphi_{\text {MPS }}$ would be adequate for representing the degrees of departure from the marginal point-symmetry model distinguishing two kinds of marginal asymmetry with respect to the midpoint.

\section{Acknowledgements}

The authors would like to thank referees for helpful comments.

\section{References}

Agresti, A. (2002). Categorical data analysis (2nd ed.). New York, NY: Wiley. doi: 10.1002/0471249688

Bhapkar, V. P. (1966). A note on the equivalence of two test criteria for hypotheses in categorical data. Journal of the American Statistical Association, 61(313), 228-235. doi: 10.1080/01621459.1966.10502021 


\section{MEASURE OF MARGINAL AVERAGE POINT-SYMMETRY}

Bishop, Y. M. M., Fienberg, S. E., \& Holland, P. W. (1975). Discrete multivariate analysis: Theory and practice. Cambridge, MA: The MIT Press.

Bowker, A. H. (1948). A test for symmetry in contingency tables. Journal of the American Statistical Association, 43(244), 572-574. doi:

10.1080/01621459.1948.10483284

Caussinus, H. (1965). Contribution à l'analyse statistique des tableaux de correlation [Contribution to the statistical analysis of correlation tables]. Annales de la Faculté des Sciences de Toulouse Série 4, 29(1966), 77-182. doi:

10.5802/afst.519

Goodman, L. A. (1979). Multiplicative models for square contingency tables with ordered categories. Biometrika, 66(3), 413-418. doi:

10.1093/biomet/66.3.413

McCullagh, P. (1978). A class of parametric models for the analysis of square contingency tables with ordered categories. Biometrika, 65(2), 413-418. doi: 10.1093/biomet/65.2.413

Stuart, A. (1955): A test for homogeneity of the marginal distributions in a two-way classification. Biometrika, 42(3-4), 412-416. doi: 10.1093/biomet/42.34.412

Tahata, K., \& Tomizawa, S. (2014). Symmetry and asymmetry models and decompositions of models for contingency tables. SUT Journal of Mathematics, 50(2), 131-165. doi: 10.20604/00000822

Tomizawa, S. (1985). The decompositions for point-symmetry models in two-way contingency tables. Biometrical Journal, 27(8), 895-905. doi: 10.1002/bimj.4710270811

Tomizawa, S. (1994). Two kinds of measures of departure from symmetry in square contingency tables having nominal categories. Statistica Sinica, 4(1), 325-334. Retrieved from http://www3.stat.sinica.edu.tw/statistica/j4n1/j4n120/j4n120.htm

Tomizawa, S. (1995). Measures of departure from marginal homogeneity for contingency tables with nominal categories. Journal of the Royal Statistical Society. Series D (The Statistician), 44(4), 425-439. doi: 10.2307/2348892

Tomizawa, S., Miyamoto, N., \& Hatanaka, Y. (2001). Theory \& methods: Measure of asymmetry for square contingency tables having ordered categories. The Australian and New Zealand Journal of Statistics, 43(3), 335-349. doi: 10.1111/1467-842x.00180 


\section{IKI \& TOMIZAWA}

Tomizawa, S., Seo, T., \& Yamamoto, H. (1998). Power-divergence-type measure of departure from symmetry for square contingency tables that have nominal categories. Journal of Applied Statistics, 25(3), 387-398. doi:

10.1080/02664769823115

Tomizawa, S., \& Tahata, K. (2007). The analysis of symmetry and asymmetry: orthogonality of decomposition of symmetry into quasi-symmetry and marginal symmetry for multi-way tables. Journal de la Société Française de Statistique, 148(3), 3-36. Retrieved from http://www.numdam.org/item/JSFS_2007_148_3_3_0/

Tomizawa, S., Yamamoto, K., \& Tahata, K. (2007). An entropy measure of departure from point-symmetry for two-way contingency tables. Symmetry: Culture and Science, 18(4), 279-297.

Wall, K. D., \& Lienert, G. A. (1976). A test for point-symmetry in Jdimensional contingency-cubes. Biometrical Journal, 18, 259-264.

Yamamoto, K., Tahata, K., Suzuki, M., \& Tomizawa, S. (2011). Measure of departure from marginal point-symmetry for two-way contingency tables. Statistica, 71(3), 367-380. Retrieved from https://rivistastatistica.unibo.it/article/view/3620 


\section{MEASURE OF MARGINAL AVERAGE POINT-SYMMETRY}

\section{Appendix 1}

2 For an $R \times C$ contingency table with ordered categories, the measure of departure

3 from the marginal point-symmetry model considered by Yamamoto et al. (2011) is

4 given as follows: assuming that $\left\{p_{i \cdot}+p_{i^{*}} \neq 0\right\}$ and $\left\{p \cdot j+p \cdot j^{* *} \neq 0\right\}$, for $\lambda>-1$,

5

6

7

8 where

9

10

14

$$
\psi_{1}^{(\lambda)}=\frac{1}{2^{\lambda}-1} \sum_{i=1}^{[R / 2]}\left[q_{i \cdot}\left\{\left(\frac{q_{i \cdot}}{q_{i \cdot}^{\mathrm{MPS}}}\right)^{\lambda}-1\right\}+q_{i^{*} \cdot}\left\{\left(\frac{q_{i^{*}}}{q_{i \cdot}^{\mathrm{MPS}}}\right)^{\lambda}-1\right\}\right],
$$

15

16 and the value at $\lambda=0$ is taken to be the limit as $\lambda \rightarrow 0$. Note that (i) $0 \leq \psi^{(\lambda)} \leq 1$, 17 (ii) $\psi^{(\lambda)}=0$ if and only if the marginal point-symmetry model holds, and (iii) $\psi^{(\lambda)}=1$

18 if and only if $p_{i}=0$ (then $p_{i^{*} \cdot}>0$ ) or $p_{i^{*}}=0$ (then $p_{i \cdot}>0$ ) for $i=1, \ldots,[R / 2]$ and

$19 p \cdot j=0\left(\right.$ then $\left.p \cdot j^{* *}>0\right)$ or $p \cdot j^{* *}=0($ then $p \cdot j>0)$ for $j=1, \ldots,[C / 2]$.

20 The sample version of $\psi^{(\lambda)}, \hat{\psi}^{(\lambda)}$, is given by $\psi^{(\lambda)}$ with $\left\{p_{i j}\right\}$ replaced by $\left\{\hat{p}_{i j}\right\}$.

21 Yamamoto et al. (2011) also gave that $\sqrt{n}\left(\hat{\psi}^{(\lambda)}-\psi^{(\lambda)}\right)$ has asymptotically (as $22 n \rightarrow \infty)$ a normal distribution with mean zero and variance 


\section{IKI \& TOMIZAWA}

1

$$
\sigma^{2}\left[\psi^{(\lambda)}\right]=\frac{1}{\left(\delta_{1}+\delta_{2}\right)^{2}} \sum_{i=1}^{R} \sum_{j=1}^{C}\left(w_{i j}^{(\lambda)}-d_{i j} \psi^{(\lambda)}\right)^{2} p_{i j},
$$

3

4 where $\sigma^{2}\left[\psi^{(0)}\right]=\lim _{\lambda \rightarrow 0} \sigma^{2}\left[\psi^{(\lambda)}\right]$, and

5

6

$$
\begin{aligned}
w_{i j}^{(\lambda)} & =d_{1(i)} \psi_{1}^{(\lambda)}+\delta_{1} \Delta_{1(i)}^{(\lambda)}+d_{2(j)} \psi_{2}^{(\lambda)}+\delta_{2} \Delta_{2(j)}^{(\lambda)}, \\
d_{i j} & =d_{1(i)}+d_{2(j)},
\end{aligned}
$$

7

8 with

9

10

$$
d_{1(i)}= \begin{cases}0 & R \text { odd and } i=\frac{R+1}{2} \\ 1 & \text { otherwise }\end{cases}
$$

$$
d_{2(j)}= \begin{cases}0 & C \text { odd and } j=\frac{C+1}{2} \\ 1 & \text { otherwise }\end{cases}
$$

11

12 and, for $\lambda>-1, \lambda \neq 0$,

13

14

$$
\Delta_{1(i)}^{(\lambda)}=\left\{\begin{array}{lr}
0 & R \text { odd, } i=\frac{R+1}{2}, \\
\frac{1}{\delta_{1}}\left[1-\psi_{1}^{(\lambda)}-\frac{2^{\lambda}}{2^{\lambda}-1}\left\{1-\left(q_{i \cdot}^{c}\right)^{\lambda}-\lambda q_{i^{*}}^{c}\left(\left(q_{i .}^{c}\right)^{\lambda}-\left(q_{i^{*}}^{c}\right)^{\lambda}\right)\right\}\right] & \text { otherwise, }
\end{array}\right.
$$

$$
\Delta_{2(j)}^{(\lambda)}=\left\{\begin{array}{lr}
0 & C \text { odd }, j=\frac{C+1}{2} \\
\frac{1}{\delta_{2}}\left[1-\psi_{2}^{(\lambda)}-\frac{2^{\lambda}}{2^{\lambda}-1}\left\{1-\left(q_{\cdot j}^{c}\right)^{\lambda}-\lambda q_{\cdot j^{* *}}^{c}\left(\left(q_{\cdot j}^{c}\right)^{\lambda}-\left(q_{\cdot j^{* *}}^{c}\right)^{\lambda}\right)\right\}\right] \text { otherwise, }
\end{array}\right.
$$

15

16

$$
q_{i .}^{c}=\frac{q_{i} .}{q_{i \cdot}+q_{i^{*}} .}, \quad q_{i^{*} \cdot}^{c}=\frac{q_{i^{*}} .}{q_{i \cdot}+q_{i^{*}} .} \quad\left(i=1, \ldots,\left[\frac{R}{2}\right]\right),
$$

$$
q_{\cdot j}^{c}=\frac{q_{\cdot j}}{q_{\cdot j}+q_{\cdot j^{* * *}}}, \quad q_{\cdot j^{* * *}}^{c}=\frac{q_{\cdot j^{* * *}}}{q_{\cdot j}+q_{\cdot j^{* *}}} \quad\left(j=1, \ldots,\left[\frac{C}{2}\right]\right) .
$$




\section{MEASURE OF MARGINAL AVERAGE POINT-SYMMETRY}

\section{$1 \quad$ Appendix 2}

2 The code is available in $\mathrm{R}$. The data matrix formed by observed frequencies should

3 be an $r \times c$ matrix named $\mathbf{m}$. The function mod.MPS gives a result of a $3 \times 4$ matrix,

4 including estimated measure, standard error, and 95\% Confidence interval for three

5 kinds of measures.

6

7 file $<-$ "table"

$8 \mathrm{~m}<-$ read.table(paste(file, ".txt", sep=" "))

$9 \quad \mathrm{~N}<-\operatorname{sum}(m)$

$10 \quad r<-\operatorname{nrow}(m)$

11 c $<-n \operatorname{ncol}(m)$

$12 \mathrm{p}<-\mathrm{m} / \mathrm{N}$

13

14

15

16

17

18

19

20

21

22

23

24

25

26

27

28

29

30

31

mod.MPS $<-$ function ()\{

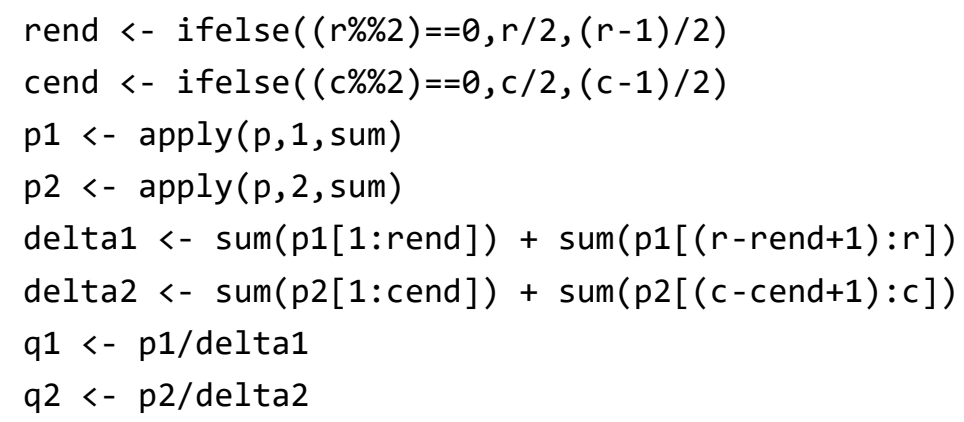

36 


\section{IKI \& TOMIZAWA}

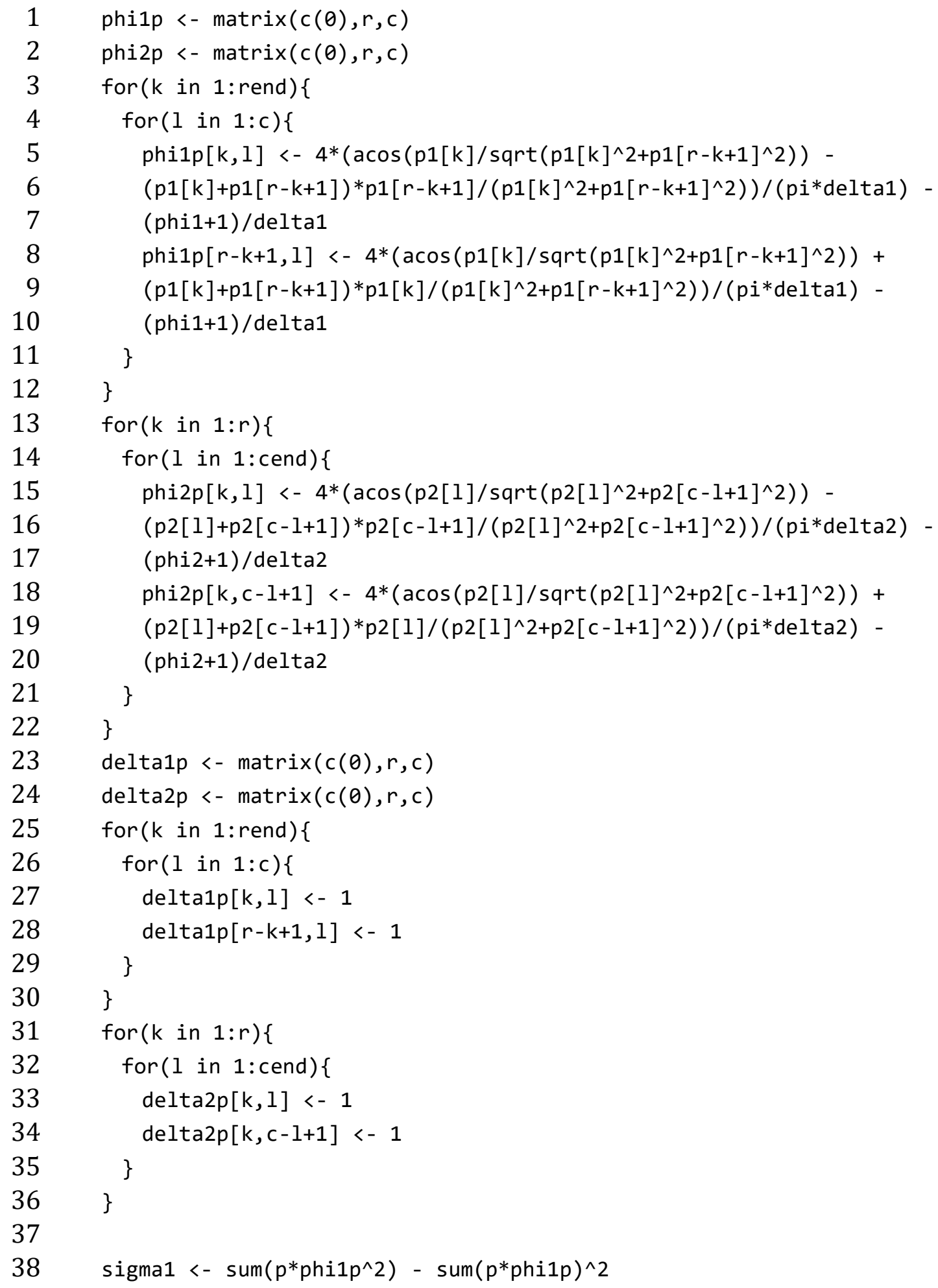




\section{MEASURE OF MARGINAL AVERAGE POINT-SYMMETRY}

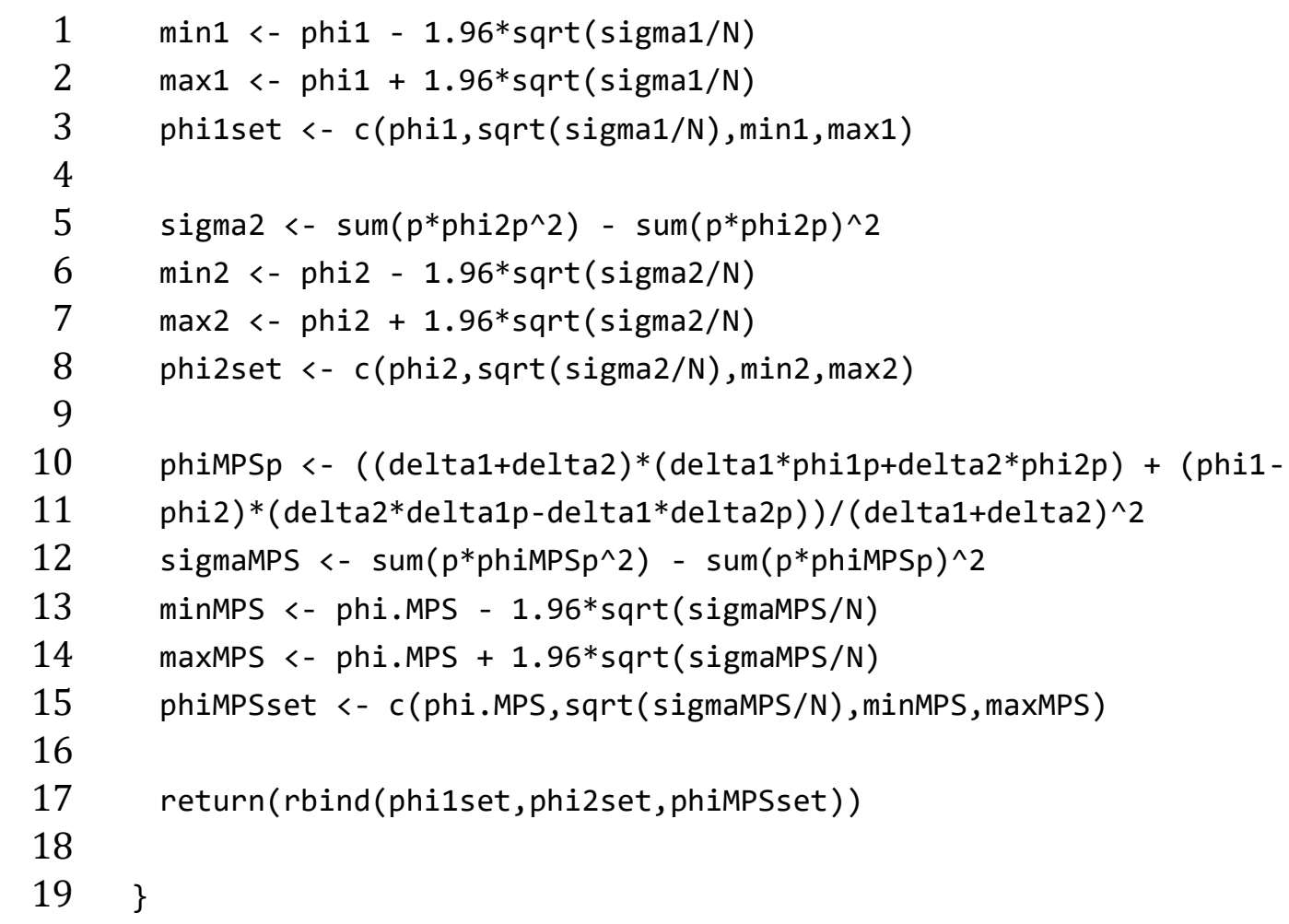

